\title{
Soft-x-ray interferometer for single-shot laser linewidth measurements
}

\author{
Juan L. A. Chilla and Jorge J. Rocca \\ Department of Electrical Engineering, Colorado State University, Fort Collins, Colorado 80523
}

Oscar E. Martinez and Mario C. Marconi

Departamento de Física, Universidad de Buenos Aires, 1428 Buenos Aires, Argentina

Received January 23, 1996

\begin{abstract}
A soft-x-ray Mach-Zehnder interferometer configuration that makes use of the time delay introduced by diffraction gratings to conduct single-shot measurements of the linewidth of soft-x-ray laser amplifiers is proposed and analyzed. The scheme was experimentally demonstrated in the near-IR region of the spectrum by measurement of the mode separation of a semiconductor laser. A symmetric configuration with compensated time delays that can be implemented for plasma diagnostics and for evaluating soft-x-ray optics is also discussed. () 1996 Optical Society of America
\end{abstract}

There is significant interest in the measurement of the linewidths of soft-x-ray lasers and their variation with amplification, because of the linewidths' fundamental importance to gain, radiative transport, and amplifier performance. ${ }^{1,2}$ The profile of unamplified plasma lines is the result of Doppler broadening, Stark broadening, and collisional rates and can also be influenced by effects such as plasma turbulence. ${ }^{3}$ Radiation transport also can strongly influence the line profile, as reabsorption broadens the line. In the case of amplified lines the line profiles narrow as a function of plasma length in the nonsaturated amplification regime, and they can rebroaden later as the amplification saturates if the intrinsic profiles of the lines are dominated by inhomogeneous broadening. These measurements are, however, difficult to perform, because of the very high resolution required, typically $\Delta \lambda / \lambda<10^{-4}$. Recently the first linewidth measurements of amplified soft-x-ray laser lines were performed at Lawrence Livermore National Laboratory. ${ }^{2}$ In those experiments the line profiles of some of the soft-x-ray laser lines pumped by the Nova laser facility were measured with a sophisticated highresolution grazing-incidence spectrograph, with a distance of more than $8.5 \mathrm{~m}$ between the laser plasma and the detector plane. Although such instruments have a resolution of approximately 30000 , their cost and size can make them inaccessible to many of the smaller research groups currently involved in the development of compact tabletop soft-x-ray laser sources. ${ }^{4-7}$

The high intensity of soft-x-ray lasers makes it possible to perform high-resolution line profile measurements by use of interferometric techniques. Interferometric techniques have been widely used to measure the linewidths of visible lasers. However, interferometric measurement of line profiles in the soft-x-ray regime is significantly more difficult. A soft-x-ray interferometer that uses thin-film multilayer beam splitters has been implemented at Lawrence Livermore National Laboratory and has been demonstrated in the diagnostics of dense laser- created plasmas. ${ }^{8}$ Recently a linewidth measurement of the neonlike yttrium x-ray laser was performed interferometrically; the decay of the contrast of the interference fringes was measured as a function of the detuning of the length of one of the arms of the interferometer for a sequence of soft-x-ray laser shots. ${ }^{9}$

In this Letter we propose and investigate a novel soft-x-ray interferometer design that takes advantage of the time delay introduced by diffraction gratings to conduct single-shot measurements of the soft-x-ray laser line profiles. The lateral delay introduced by gratings was previously used for the construction of single-shot autocorrelators for the measurement of ultrashort pulses. ${ }^{10}$ The new type of compact $(<1 \mathrm{~m}$ in length) Mach-Zehnder interferometer that we propose has a resolution of 90000 when constructed with 5-cm-wide gratings of 600 grooves $/ \mathrm{mm}$. It has the advantages of allowing one to perform line profile measurements in a single shot and of not requiring the use of sophisticated x-ray beam splitters. It also can be constructed with off-the-shelf gratings, in which all the reflections are at grazing incidence, with the consequent high throughput without the need of special coatings. A visible Mach-Zehnder interferometer that uses gratings as beam splitters has been used to test fine-ground surfaces at grazing incidence. ${ }^{11}$ The design of a soft-x-ray Smartt interferometer that uses a grating to improve efficiency was recently discussed by Bokor et al. ${ }^{12}$

The layouts of two possible configurations of the proposed interferometer are depicted in Fig. 1. Both configurations ( $a$ and $b$ ) consist of two flat gratings (G1 and G2) acting as beam splitters in a Mach-Zehnder arrangement. In both cases the beams are redirected by reflections in mirrors (M1-M4) so that after diffraction on the second gratings they emerge collinearly and the astigmatism of the system is compensated. The interference pattern produced can be recorded in a twodimensional CCD detector. One can obtain interference fringes in any desired density and parallel to the 

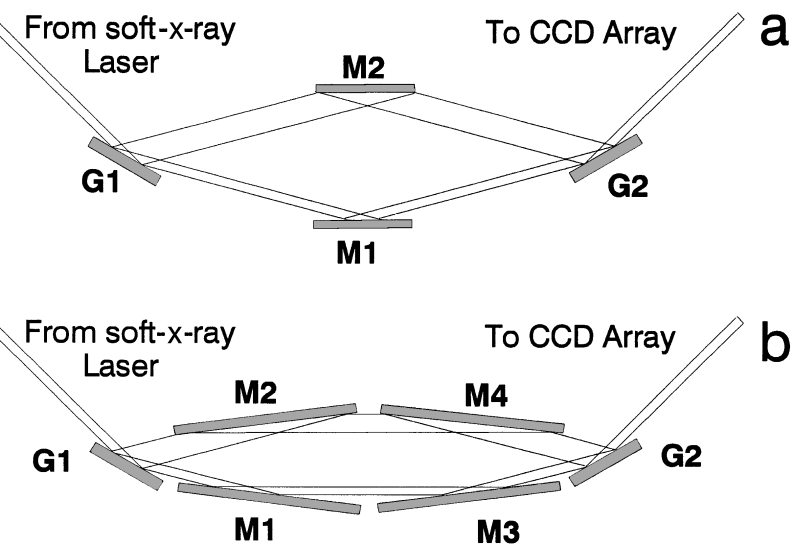

Fig. 1. Proposed Mach-Zehnder interferometer configurations with gratings G1 and G2 acting as beam splitters. Configurations a, with compensated delay, and b, with noncompensated delay, are shown.

plane of the system by introducing a slight inclination in the perpendicular direction. On incidence upon grating G1, the x-ray laser beam is diffracted into different orders. By properly choosing the incidence angle and blaze of the grating, one can ensure that most of the incoming energy is split evenly between the zero and the first diffraction orders.

As can be readily shown with geometric considerations, light diffracted in the first order acquires a transverse-position-dependent delay. In the simplest configuration depicted in Fig. 1a, there is one reflection in each branch. The consequent inversion experienced by the beams compensates for the delays introduced by G1 and G2. One can adapt this configuration to conduct plasma diagnostics or to test soft-x-ray optics by introducing the sample into the optical path of one branch and using the other branch as a reference. In the second configuration the delays introduced by the gratings are not compensated. When additional inversion (reflection) is introduced into the first-order branch of the interferometer the delays introduced by both gratings add up, so that the interfering beams have a relative delay that depends on the transverse position on the detector. An extra reflection is also necessary in the zero-order branch (Fig. 1b) because of the low spatial coherence of existing x-ray lasers. Any vertical (out of the plane of the page) cross section will show the typical oscillatory behavior of two interfering light beams, but the visibility of the fringes will depend on the horizontal (in-plane) position on the detector. Close to the center of the beam, where both paths are equal, the visibility will be maximum, and toward the edges of the detector the visibility will drop because of the finite coherence length (inverse linewidth) of the laser source. The line profile can be obtained as the Fourier transform of the delay dependence of the visibility of the fringes. ${ }^{13}$ If several laser lines are emitting, their effect will be evident as modulations in the delay dependence of the visibility. All the spectral information is obtained in a single shot of the laser. The calibration of the path difference $\Delta z$ of the system is given by $\Delta z=2 x \lambda /(d \cos \gamma)$, where $x$ denotes the distance measured on the detector plane, $d$ is the groove spacing, and $\gamma$ is the incidence angle on the first grating. A conservative estimate of the resolution is obtained when we require that the visibility decay to $1 / e$ of its maximum value within the range of delay of the system. The resulting resolution depends on the grating size $a$ and is given by $\Delta \lambda / \lambda=d /(\pi a)$. Because of the inverse Fourier relationship, the spatial resolution of the detector does not limit the resolution but instead puts a limit on the maximum linewidth observable with the interferometer.

To demonstrate the feasibility of the aparatus, experiments were conducted in the near-IR region of the spectrum by use of a vertical-cavity surfaceemitting laser diode, ${ }^{14}$ which could be adjusted to emit in two modes of similar amplitude. Using standard 1-in. $(2.54 \mathrm{~cm})$ gratings of 600 grooves $/ \mathrm{mm}$ made it possible to measure the spectral separation of the modes of the available laser, but the spectral width of the individual modes was not resolved because of $\sim 1$-m coherence length. Interferograms were obtained for both the compensated (Fig. 2a) and the noncompensated (Fig. $2 \mathrm{~b}$ ) configurations by the use of collimated beams of approximately $3 \mathrm{~mm}$ FWHM. As can be seen from Fig. 2a, the visibility of the fringes is roughly constant across the entire pattern, dropping only toward the edges of the illuminated region.

The interferogram shown in Fig. $2 \mathrm{~b}$ was obtained with the configuration depicted in Fig. $1 \mathrm{~b}$ and shows a transverse-position dependence caused by the delay introduced by the gratings. The dark and the light regions of the interferogram can be seen to alternate as the visibility of the fringes is modulated as a function of the position on the detector. The transverse-position period of the visibility, $1.25 \mathrm{~mm}$, translates into a delay of $1.94 \mathrm{~mm}$ after the measured central wavelength of $0.856 \mu \mathrm{m}$ and the incidence angle of $48.7^{\circ}$ are taken into account. This delay corresponds to a spectral separation of $(0.377 \pm 0.018) \mathrm{nm}$, which is in excellent agreement with the independently measured emission spectrum of the laser, which gave a separation of $(0.371 \pm 0.010) \mathrm{nm}$.

For measurement of the linewidths of soft-x-ray lasers, consideration also has to be given to their poor spatial coherence. Radiation going through different
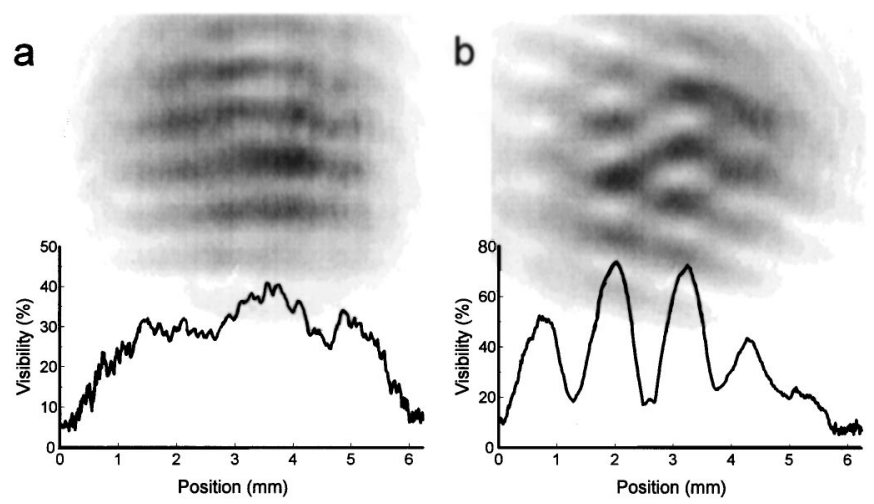

Fig. 2. Interferograms obtained from a multimode laser diode with a, the configuration in Fig. 1a, where the visibility of the fringes is roughly constant across the pattern, and $b$, with the configuration in Fig. $1 b$. The visibility of the fringes oscillates with a period corresponding to the spectral separation of the modes. 
arms of the interferometer after splitting at the first grating should fall upon points on the plane of the detector that are within the coherence distance, which in existing $\mathrm{x}$-ray lasers can be $\sim 100-200 \mu \mathrm{m}$ at distances of $2-3 \mathrm{~m}$ from the laser source. ${ }^{9}$ Factors contributing to difference paths in both arms are the dispersion of different frequencies in the gratings at first order and the lateral magnification experienced by the beam as it goes through the first-order branch of the interferometer. A simple ray-tracing analysis shows that radiation detuned from the central frequency by $\Delta \lambda$ and propagating at an angle $\Delta \varphi$ arrives at points on the detector separated by a distance $\Delta x$, given by

$$
\Delta x=\Delta \lambda\left(\frac{z \cos ^{2} \gamma}{\cos ^{2} \theta}+2 z_{d}\right)-\Delta \varphi z\left(1-\frac{\cos ^{2} \gamma}{\cos ^{2} \theta}\right),
$$

where $\theta$ is the diffracted angle from the first grating, $z$ is the distance between the gratings, and $z_{d}$ is the distance from the second grating to the detector. For the system to work properly $\Delta x$ should be smaller than the coherence distance of the source. For a grazing incidence configuration $\cos \theta>\cos \gamma$, and the first term is dominated by the dispersion after the second grating, which is minimized because the detector is located as close as possible to the grating. The same relation between the angles makes the expression in parentheses in the second term close to unity, and thus the only way of minimizing the second term is by using a fairly well-collimated beam. A beam divergence of approximately $0.2 \mathrm{mrad}$ would be required for an inteferometer with $50-\mathrm{cm}$ spacing between gratings. This can be achieved by means of a curved mirror between the soft-x-ray laser and the interferometer.

Below we present the design of the proposed interferometer for measurement of the linewidth of the 46.9-nm laser line of neonlike argon. Such a transition is of interest in relation to the recent observation of large amplification in a discharge-created plasma. ${ }^{4}$ Following the previous discussion, a resolution of close to 50000 can be attained with a standard grating of $5 \mathrm{~cm}$ in size and 300 grooves $/ \mathrm{mm}$. If the incidence angle is chosen to be $86^{\circ}$, the calibration results in $405 \mu \mathrm{m}$ of delay per millimeter on the detector for a total delay range of $1.4 \mathrm{~mm}$ on the $3.5-\mathrm{mm}$-wide illuminated area. Radiation with $\Delta \lambda / \lambda=5 \times 10^{-5}$ at $46.9 \mathrm{~nm}$ would produce in that width fringes with visibility decaying to $e^{-2}$ to both sides of the central maximum. For maximum fringe visibility the blaze of the grating should be $1.6^{\circ}$. The dimensions of the interferometer are determined by the size of the folding mirrors. Because of the grazing incidence angles and the magnification experienced by the first-order branch, the mirrors have a minimum required size of $32 \mathrm{~cm}$. The total size of the instrument is nevertheless compact, and the interferometer can be constructed in a vacuum chamber of $\sim 75 \mathrm{~cm} \times 15 \mathrm{~cm}$.

In summary, we propose a new type of MachZehnder interferometer that makes use of the transverse-position-dependent delay introduced by diffraction gratings to conduct linewidth measurements on soft-x-ray lasers in a single shot. The transverse-position-dependent delay introduced by the gratings can be compensated for use of the interferometer for plasma diagnostics and testing of high-quality optics. The proposed interferometer design was analyzed theoretically, with the beam divergence and spatial coherence taken into account, and was experimentally demonstrated in the IR region of the spectrum with a diode laser of known spectral characteristics.

This research was supported by National Science Foundation grant ECS-9401952 and Fundación Antorchas proyecto A13218/1. The support of the Colorado Advanced Technology Institute for collaboration with Hyperfine (Boulder, Colo.) is also acknowledged. O. E. Martinez and M. C. Marconi are members of the staff of the Consejo Nacional de Investigaciones Científicas y Técnicas de la República Argentina.

\section{References}

1. J. A. Koch, B. J. MacGowan, L. B. Da Silva, D. L. Matthews, J. H. Underwood, P. J. Batson, and S. Mrowka, Phys. Rev. Lett. 68, 3291 (1992).

2. J. A. Koch, B. J. MacGowan, L. B. Da Silva, D. L. Matthews, J. H. Underwood, P. J. Batson, R. W. Lee, R. A. London, and S. Mrowka, Phys. Rev. A 50, 1877 (1994).

3. H. R. Griem, Plasma Spectroscopy (McGraw-Hill, New York, 1964).

4. J. J. Rocca, V. Shlyaptsev, F. G. Tomasel, O. D. Cortazar, D. Hartshorn, and J. L. A. Chilla, Phys. Rev. Lett. 73, 2192 (1994).

5. B. Lemoff, G. Y. Yin, C. L. Gordon III, C. P. J. Barty, and S. E. Harris, Phys. Rev. Lett. 74, 1574 (1995).

6. P. V. Nickles, M. Schmurer, M. P. Kalashnikov, I. Will, W. Sanders, and V. N. Shlyaptsev, Proc. SPIE 2520, 373 (1995); A. Morozov, L. Polansky, and S. Suckewer, Proc. SPIE 2520, 373 (1995).

7. S. Basu, P. L. Hagelstein, and J. G. Goodberlet, Appl. Phys. B 57, 303 (1993).

8. L. B. Da Silva, T. W. Barbee, Jr., R. Cauble, P. Celliers, D. Ciarlo, S. Libby, R. A. London, D. Matthews, S. Mrowka, J. C. Moreno, D. Ress, J. E. Trebes, and A. Wan, Phys. Rev. Lett. 74, 3991 (1995).

9. P. Celliers, F. Weber, L. B. Da Silva, T. W. Barbee, Jr., R. Cauble, A. S. Wan, and J. C. Moreno, Opt. Lett. 20, 1907 (1995).

10. R. Wyatt and E. E. Marinero, Appl. Phys. 25, 297 (1981).

11. P. Hariharan, Opt. Eng. 14, 257 (1975).

12. J. Bokor, K. A. Goldberg, H. Medecki, R. Beguiristain, K. Jackson, D. T. Attwood, G. E. Sommargren, R. Hostetler, and J. P. Spallas, presented at the Optical Society of America Annual Meeting, Portland, Ore. September 10-15, 1995.

13. M. Born and E. Wolf, Principles of Optics, 2nd ed. (Pergamon, Oxford, 1964).

14. J. L. A. Chilla, B. Benware, M. E. Watson, P. Stanko, J. J. Rocca, C. Wilmsen, S. Feld, and R. Leiberguth, IEEE Photon. Technol. Lett. 7, 449 (1995). 